\section{Glioblastoma multiforme subterfuge as acute cerebral hemorrhage: A case report and literature review}

\author{
Seidu A. Richard, 1-3 Yunxia Ye, ${ }^{4}$ \\ Hao Li, ${ }^{1}$ Lu Ma, ${ }^{1}$ Chao You ${ }^{1}$ \\ 1 Department of Neurosurgery, Post \\ Graduate Training Centre, West China \\ Hospital, Sichuan University, China; \\ 2Department of Immunology, Jiangsu \\ University, China; ${ }^{3}$ Department of \\ Surgery, Volta Regional Hospital, Ho, \\ Ghana; ${ }^{4}$ Department of Pathology, West \\ China Hospital, Sichuan University, \\ China.
}

\begin{abstract}
Hemorrhagic related Glioblastoma multiforme (GBM) are rare and characterizes with severe clinical scuffle. The etiology of this presentation although not well known is believed to be multifactorial. We present a case as well as review on the pathogenesis of evolution of the hematoma into ring enhancing features of GBM on imaging studies. We present a case of 28 years old man who suddenly went into coma for 9 hours preceded with seizures that latest for 10 minutes. He had no focal neurological signs. CT-Scans images indicated acute cerebral hemorrhage near the frontal horn of the left ventricle with brain edema about the hemorrhagic lesion and MRI done a week later revealed a cerebral ring enhancing lesion. The lesion was partially resected during surgery and immunohistochemical staining confirmed GBM (WHO, grade 4). The diagnosis of intratumoral hemorrhage in GBM was very challenging at the initial stages but with time the hematoma evolved into ring enhancing images typical of GBM. It's not every intracranial hematoma that is of pure vascular origin.
\end{abstract}

\section{Introduction}

Hemorrhagic related Glioblastoma multiformes (GBMs) are rare and characterizes with severe clinical challenges. ${ }^{1-3}$ GBM is the most common and lethal type of astrocyte-derived tumor, constituting $50 \%$ of adult primary brain tumor cases, followed by anaplastic astrocytoma which constitutes $30 \%$ and astrocytoma which also constitutes $20 \%{ }^{4}$ Licata et al. ${ }^{5}$ established that $33 \%$ of intratumoral bleed is from GBM.
The etiology of GBM-related bleeding although not well known; however, it is certainly multifactorial and factors related to this pathogenic process can be categorized into predisposing, initiating, and aggravating. ${ }^{1}$ This factors can either be local or systemic. ${ }^{1}$ While the significate local factors comprise of the state of blood vessels and capillaries, the most important systemic factor is coagulopathy which can also occur at the local level and normally maintain the bleeding process. ${ }^{1}$ The clinical manifestations of cerebral hemorrhage are not exceptionally the first clinical suggestion of a tumor and frequently convey a modest outcome.1,6-8 First hemorrhagic episode in GBM can directly lead to the death of the patient although subsequent bleeding carries a high risk. ${ }^{1}$ Therefore GBM-related hemorrhage is a very significant risk factor that can result in death of the patient. We present a case of glioblastoma that subterfuge as acute cerebral hemorrhage and review literature on GBM-related hemorrhages with more focus on the pathophysiology as well as pathogenesis of the hemorrhage.

\section{Case Report}

We present a case of 28-year-old man who suddenly went into coma for 9 hours preceded with seizures that latest for 10 minutes. He was initial managed in a periphery hospital before presenting at our facility. The seizures where tonic clonic in nature with no fever, vomiting or micturition. He had no focal neurological signs. Past medical history was unremarkable. Physical examination did not yield much. Neurological examination revealed normal cranial nerves. Power as well as reflexes were present and normal. Routine laboratory as well as other ancillary investigation were normal.

Referral images indicated acute cerebral hemorrhage near the left frontoparietal lobe extending to the frontal horn of the left ventricle with brain edema around the hemorrhagic lesion and midline shift of the brain to the right (Figure 1). The hematoma was mostly centralized at the left parietal lobe. He was admitted in the local hospital for one (1) week prior to referral. MRI done a week later at our facility revealed a cerebral ring enhancing lesion of about $4.6 \times 3.4 \times 4.0$ $\mathrm{cm}$ in diameter at the left frontoparietal lobe which appear hypo-dense on $\mathrm{Tl}$ and hyperdense on T2. On FLAIR, however the images showed slightly mixed signals with no significant enhancement after administration of contrast agent. There is significant peripheral edema and swelling of the adja-
Correspondence: $\mathrm{Lu} \mathrm{Ma}$, Department of Neurosurgery, Post Graduate Training Centre, West China Hospital, Sichuan University, 37 Guo Xue Xiang Road, Chengdu, Sichuan, 610041, P.R. China.

Tel.: +86.18980601975; Fax: +86.02885422490. E-mail:wchneuro@163.com

Key words: Glioblastoma multiforme, Hematoma, Hemorrhage, Seizures, Coagulopathy.

Contributions: SAR conceived the project, designed the study and collected patient's data. YY provided the pathology report and pictures. CY, LM, HL provided technical assistance in the study. SAR analyzed the data, prepared the illustrations and wrote the paper.

Conflict of interest: the authors declare no potential conflict of interest.

Funding: none

Received for publication: 30 December 2017. Revision received: 24 February 2018.

Accepted for publication: 1 March 2018.

This work is licensed under a Creative Commons Attribution NonCommercial 4.0 License (CC BY-NC 4.0).

(C) Copyright S.A. Richard et al., 2018

Licensee PAGEPress, Italy

Neurology International 2018; 10:7558

doi:10.4081/ni.2018.7558

cent brain tissue as well as a little blood clot at the right side of the falx. The frontal horn of the left ventricle is compressed by the lesion while the right ventricle appears normal with a slight midline shift of the falx to the right (Figure 2). The skull is normal. There is no significant increment of the lesion comparing the initial CT-Scans and the current MRI. CT-angiography done revealed stenosis of main intracranial arteries with no obvious signs of ectasia or any vascular abnormalities (Figure 3). Base on the finding above, a working diagnosis of left frontal cerebral hematoma was made to rule out neoplastic lesion because of the ring like nature of the lesion. After taking the patient and his family through a series of education and counselling surgery was scheduled.

We used the left pterion approach. After removing the bone flap, we saw a small AVM with curved nidus on the dura. Intraoperatively we saw hematoma at the left frontoparietal lobe which was mostly centralized at the parietal lobe and surrounded by a pale-yellow jelly-like tissue which was large in size measuring about $4.6 \times 3.4 \mathrm{~cm}$ with rough edges making it very 
difficult to different from the normal brain tissue. We excised a chunk of the of lesion and secured total hemostasis. The bone flap was replaced with screws and plates and the subcutaneous tissues, temporalis muscle as well as skin was closed in layers. Postoperative CT-scan revealed total evacuation of the hematoma (Figure 4). Postoperative management was uneventful. Immunohistochemical staining of sample at the pathology department revealed tumor cells that are GAFP $(+)$, Oligo2 $(+)$, EMA $(-$ ), IDH-1 (+), ATRX (+), P53 (+) and 40\% $\mathrm{Ki}-67(+)$ rate. The findings above is consistent with GBM (WHO, grade 4) (Figure 5). The sample was found to be MGMT gene methylation promoter and TERI gene mutation was seen in progress. The patient was discharged home 10 days after surgery and radiotherapy as well as chemotherapeutic sessions initiated a month after surgery. The patient is currently doing very well with no obvious complication.

\section{Discussion}

Hemorrhagic related GBMs are rare and characterizes with severe clinical scuffle. . $^{1-3}$ GBM is the most common and lethal type of astrocyte-derived tumor, constituting 50\% of adult primary brain tumor cases, followed by anaplastic astrocytoma which constitutes $30 \%$ and astrocytoma which also constitutes $20 \%{ }^{4}$ Licata et al. ${ }^{5}$ established that $33 \%$ of intratumoral bleed is from GBM, $21 \%$ from metastases, $13 \%$ from anaplastic gliomas, $12 \%$ from lowgrade gliomas, $12 \%$ from meningiomas, $5 \%$ from adenomas, $2 \%$ from hemangioblastomas, $2 \%$ from melanomas, $1 \%$ from neuroblastoma and $1 \%$ from pinealoblastoma. Kondziolka et al. 9,10 observed in their study that out 264 cases with GBM; 17 of these cases constituting about $6.4 \%$ had macroscopic hemorrhage while 34 of these cases constituting about $12.9 \%$ had microscopic hemorrhage. We also present a case of GBM that subterfuge as acute cerebral hemorrhage. The hemorrhage in our case is a mixed type although predominantly macroscopic hemorrhage.

The etiology of GBM-related bleeding is not well known; however, it is certainly multifactorial and factors related to this pathogenic process can be categorized into predisposing, initiating, and aggravating. ${ }^{1}$ Atypical new perforating blood vessels that traverse necrotic areas are believed to be the initiation points of intratumoral hemorrhage in GBM. ${ }^{11,12}$ Also, intratumoral hemorrhage in GBM can occur when large vessels are invaded by the tumor resulting in weakening and breakdown of their walls. ${ }^{11,13}$
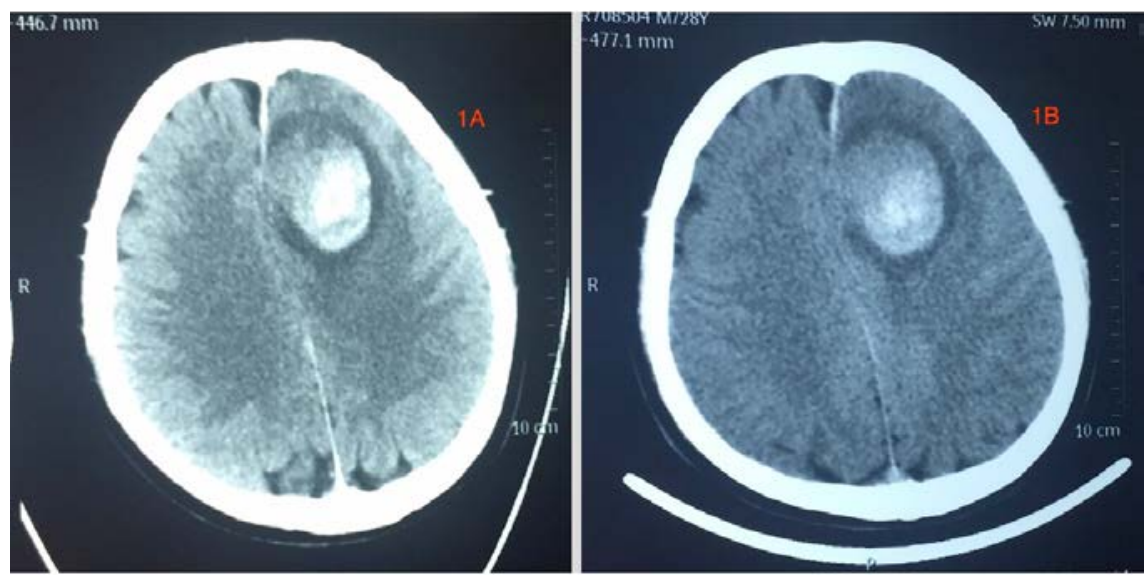

Figure 1. Referral axial CT-scan images the patient brought from the local hospital. These images show hematoma formation.
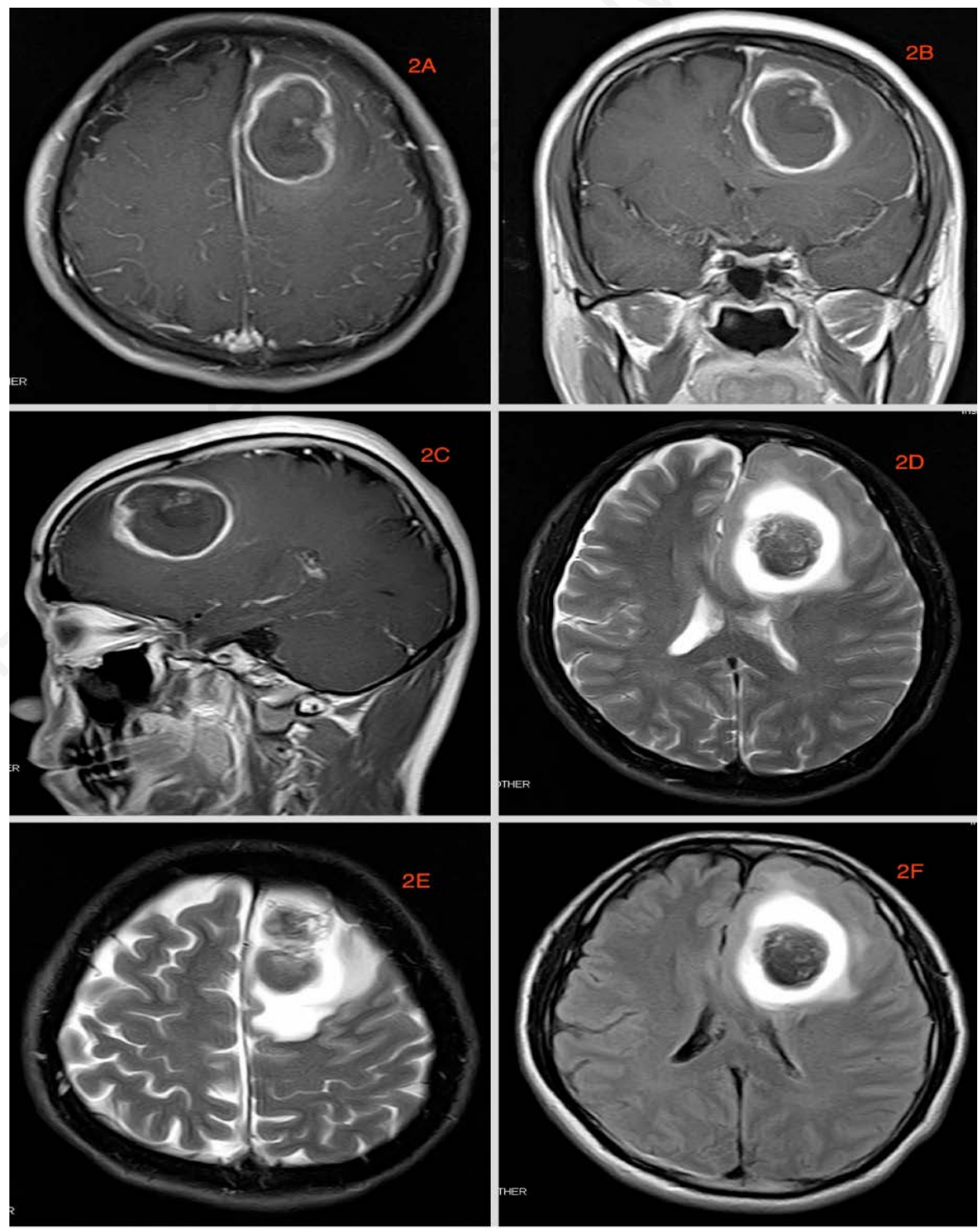

Figure 2. MRI image done at our facility. A-C) are axial, coronal and sagittal T1 image respectively. $\mathrm{D}, \mathrm{E}$ ) are axial $\mathrm{T} 2$ images while $2 \mathrm{~F}$ is FLAIR. These images show ring like lesions that are consistent with Glioblastoma. The delay window of the evolution process was up to 14 days. 
Furthermore, weak tumor vessels may not have joined the glial meshwork very well hence result in decreased resistance to the trimming forces of the brain.11,14 Nonetheless, endothelial proliferation with successive occlusion of the lumen or incidence of intratumoral arteriovenous fistulae are alternative origins of intratumoral hemorrhage. ${ }^{11,15}$ Coagulopathy which occur systemically or at the tumor level usually maintain the bleeding process. ${ }^{1}$ Therefore, making the attainment of hemostasis at hematoma very difficult if conservative treatment options are adapted or during even during surgery. ${ }^{11,16}$

The clinical manifestations of GBM can differ significantly base on the location and size of the lesion as well as the anatomic structures around the affected brain. 17-19 Headaches are quite common and unilateral with characteristically progressive in severity. 20 Considerably increased intracranial pressure may manifest as papilledema if the disease is not noticed early. ${ }^{20}$ Also, larger lesions with substantial mass effect may present with gait imbalance and urinary and/or fecal incontinence. Furthermore, depending on the location of the lesion, focal signs like hemiparesis, sensory loss, or visual field disturbances may occur and rarely, the manifestation of symptoms may be abrupt, masquerading as hemorrhagic stroke. 17,20 Nevertheless, etymological glitches may be misunderstood for confusion or delirium. ${ }^{20}$ Seizures which may have focal onset occurs in about $20-40 \%$ of cases prior to presentation. ${ }^{20,21}$ Therefore, clinically significant hemorrhages related to GBM are comparatively very rare. 1,22

Contrast radiological evaluation mostly differentiates tumors from hemorrhage because the margins between the tumors and hemorrhage is frequently welldefined.11,23 Intratumoral hemorrhage and tumor may be very difficult to differentiate during spontaneous Intracranial hemorrhage (ICH), even with contrast when the tumor is compressed by a large hemorrhage.11,24 While MRI offers enhanced tissue description and more accurately the location of lesions than CT-scan, both imaging procedures offer harmonizing evidence about intratumoral hemorrhage in GBM. 25,26 CT-scan is a faster and more readily available instrument that offers images that are simpler to interpret than MRI in patients with GBM presenting with acute intratumoral hemorrhage. Furthermore, CT-scan is extremely helpful in evaluating the distinctive sources of T1weighted hyperintensity on MRI images such as hemorrhage and calcification. ${ }^{25}$

Serial MRI studies revealed delayed evolution of hemorrhage into ring enhanc- ing lesion within a hemorrhagic GBM.6,27 This delay is probable due to the shortening of the T1 relaxation time by methemoglobin, and a magnetic predisposition consequence of intracellular hemoglobin disintegration components on the $\mathrm{T} 2$ relaxation time. Atlas et al observed a delay of 16 days from the hypointensity correlated to acute bleeding to the hyperintensity linked to the synthesis of uninhibited methemoglobin, 27 while Destian et al. observed a lengthier delay of up to numerous months. ${ }^{6}$ We also observed a delay window of up to 14 days. The pathogenesis of this delay is uncertain so further observational studies are required to finally conclude on the delay window period. Studies have shown that methemoglobin synthesis is highest at moderately
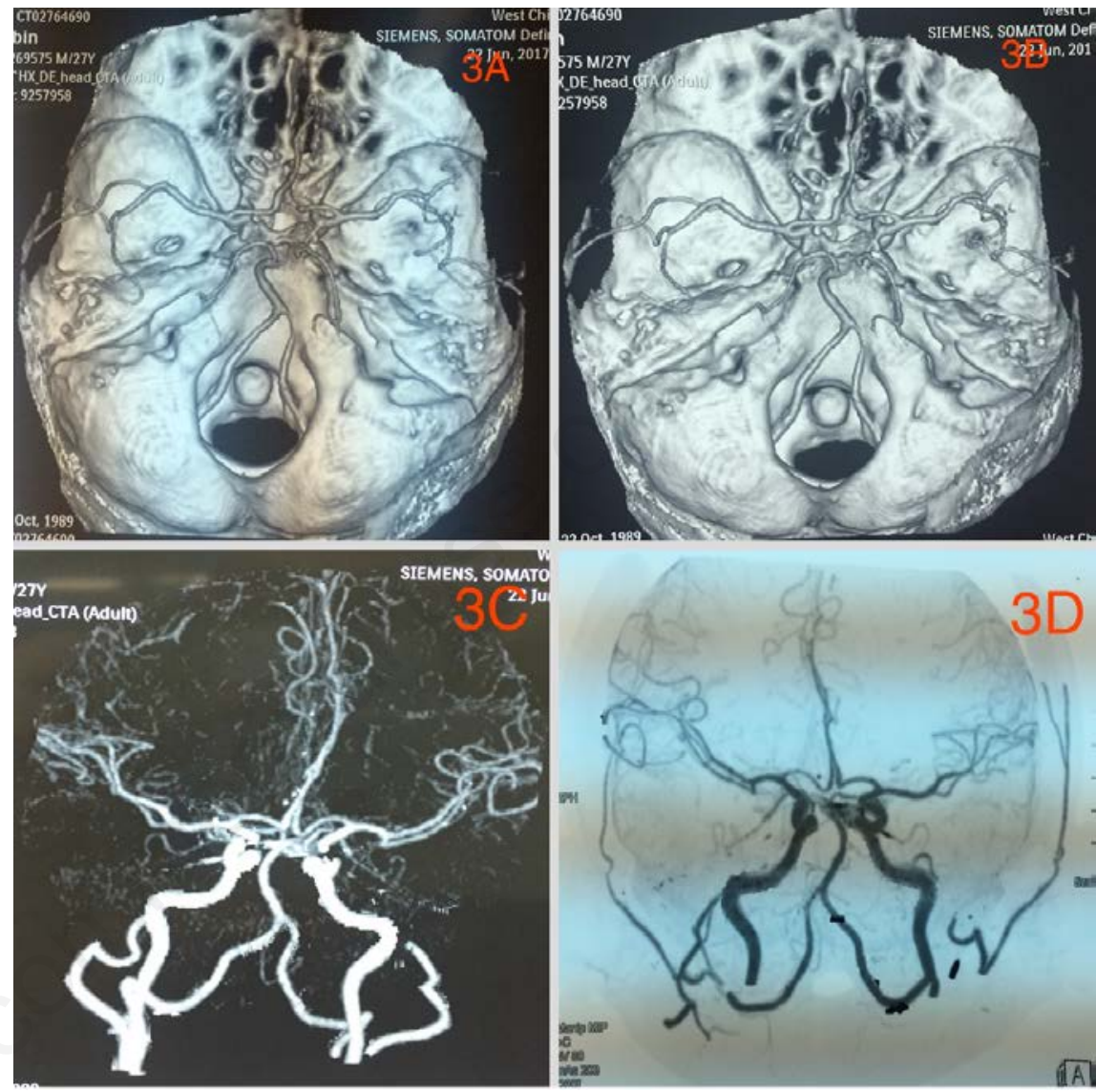

Figure 3. CT-angiography showing no vascular abnormalities.
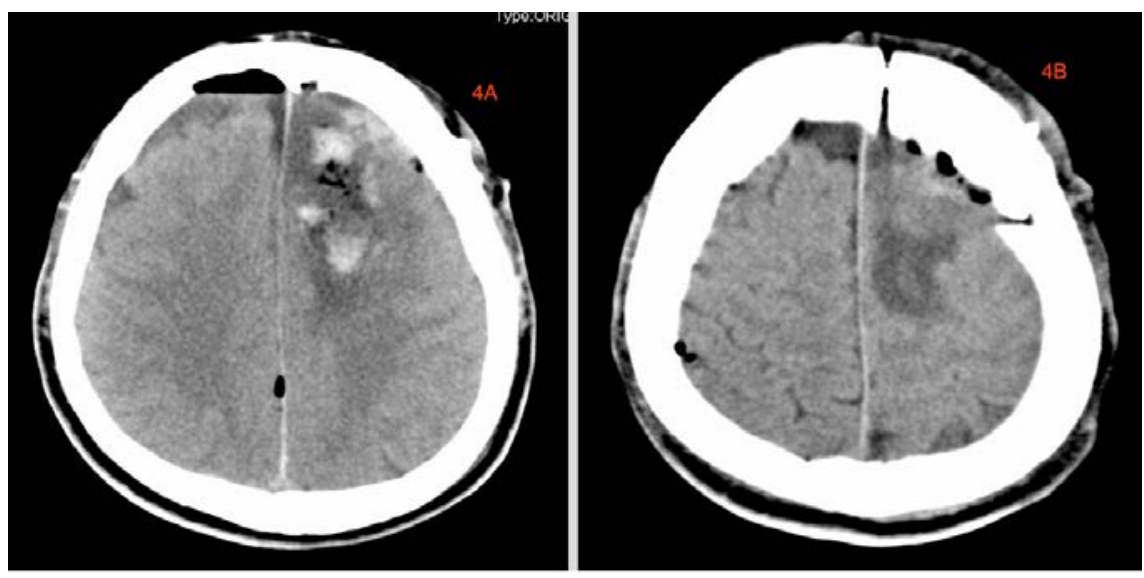

Figure 4. Postoperative CT-Scan images showing to total evacuation of the hematoma. 
low oxygen tensions of approximately 20$25 \mathrm{~mm} .6,28,29$ Subsequently, oxygen is essential for the synthesis of the mediators conscientious for the oxidation of deoxyhemoglobin to methemoglobin, besides oxygen restricting the concentration of deoxyhemoglobin availability for oxidation to methemoglobin hence the synthesis of methemoglobin is infinitesimal at zero as well as extreme oxygen tensions. ${ }^{6}$

On the other hand, studies have shown that at 20-25 mm, there is sufficient oxygen to permit the synthesis of the reducing mediators, nevertheless not sufficient to diminish the concentration of deoxyhemoglobin.6,30 Therefore, analogous hypoxia inside gliomas ought to argument instead of delay the synthesis of methemoglobin. Furthermore, the delay in evolution could be due to sustained bleeding from the tumor and/or the quick absorption of hemoglobin disintegration components by an extremely vascular glioma. It's well known that, the synthesis of methemoglobin is highest at moderately low oxygen tensions, and this be the explanation for central hyper-intensity (ring enhancing lesion) normally seen on stage-2 hemorrhagic gliomas. 6,30 Therefore, in a pure hematoma with very low oxygen tension at the center there is inhibition synthesis of reducing mediators obligatory for the oxidation of deoxyhemoglobin to methemoglobin and the synthesis of methemoglobin primarily arises from the periphery. ${ }^{6}$

The management of newly established GBM demands a multidisciplinary method and existing standard therapy comprises of near total secure surgical resection, preceded by simultaneous radiation with temozolomide (TMZ), an oral alkylating chemotherapy drug, and then adjuvant chemotherapy with TMZ. 17 GBMs are often invasive and affects eloquent areas of the brain. Most of these areas control speech, motor function, and the senses and so making far-reaching and total surgical resection difficult if not impossible. ${ }^{17}$ Furthermore, the disease succession or relapse is very common due to the extraordinary extent of invasiveness and radical surgical removal of the principal lesion is usually not beneficial since the infiltrating gliomas cells always continue to linger inside the neighboring brain. ${ }^{17,31}$ Also, modern innovations like multimodal neuronavigation and incorporation of patient-specific anatomic and functional data as well as the use of functional MRI, diffusion tensor imaging (DTI), CT-scans and MRI in preoperative planning has made surgical resection less complicated. ${ }^{17}$

Notwithstanding these technical innovations above, distinguishing normal brain and residual tumor still problematic so Zhao et al.32 advocates the use of 5-aminolevulinic acid (5-ALA) dye as a fluorescence guidance which much effective and beneficial compare to orthodox neuronavigation-guided surgery. Radiotherapy and chemotherapy are very crucial components of the management of GBM patients so after surgical resection, the patient usually waits for one month for the craniotomy wound to heal before starting these other therapeutic options. ${ }^{17}$ Radiation therapy (RT) alone after operation was the standard management regimen until 2005, when the outcome of a pivotal phase III trial transformed this peculiar management regimen for GBM. This trial also known as the Stupp regimen, 17,33 demonstrated adequately that external beam of RT with simultaneous TMZ chemotherapy is more efficacious compare to RT alone.

It is worth emphasizing that multiple potential long-term hitches of whole-brain

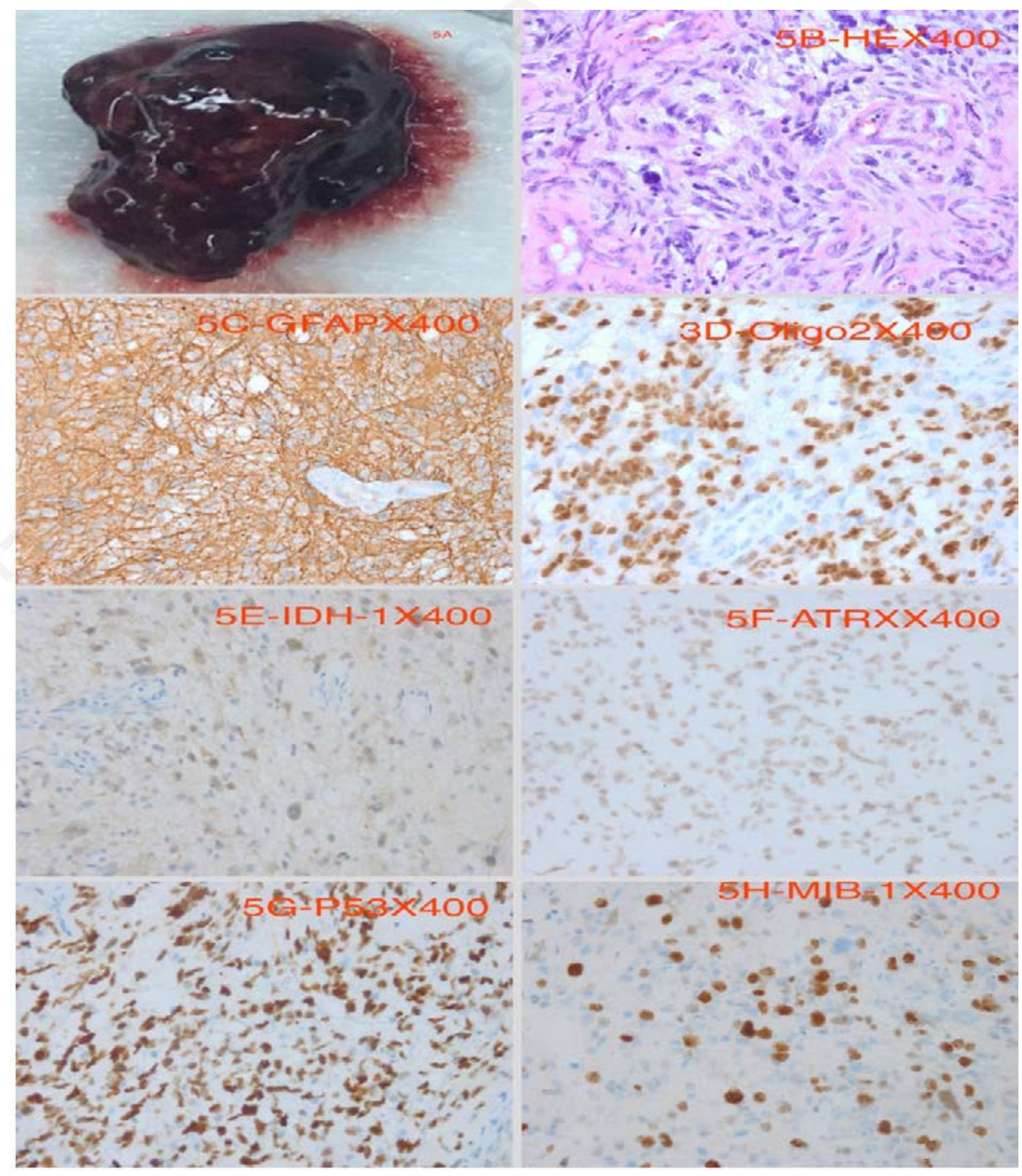

Figure 5. Pathological images. A) Is the evacuated hematoma, while B-H) are images of varies immunohistochemical staining. B) HE 400 $\times$, C) GFAP 400×, D) Oligo2 400 $\times$, E) IDH-1 400×, F) ATRX 400×, G) P53 400×, H) MIB-1 400×.
RT (WBRT) such as endocrinopathy, neurocognitive toxicity, and RT-induced leukoencephalopathy has limited the use of WBRT in the management of newly detected GBM. These grave hitches have led to further investigate into the use of involved field RT (IFRT) to minimize toxicity. ${ }^{17}$ Narayana indicated that IFRT dispenses external beam of RT to the tumor with a 2$3 \mathrm{~cm}$ perimeter with assumption that that after RT, ${ }^{34} \mathrm{GBM}$ relapses within $2 \mathrm{~cm}$ of the primary tumor location in $80-90 \%$ of patients. Further studies 17,34 have proven that the use of three-dimensional conformal beam of RT or intensity-modulated RT is the gold standard treatment and mainstream total dose of 60 Gy in 1.8-2 Gy fractions must be delivered five days per week for six weeks. Barani \& Larson $^{35}$ indicated that with RT doses to $60 \mathrm{~Gy}$, patient have a more survival rate but with increased doses above this may lead to augmented toxicity with no added survival advantages. 
The morphologically of GBM comprises of small spindle-shaped cells, characterized by polymorphism, anaplasia and distinct anisokaryosis with acidophilic cytoplasm and unclear cellular margins. ${ }^{36}$ They have oval or elongated nuclei with coarsely clumped hyperchromatic chromatin and multiply distinct nucleoli situated centrally or peri-centrally with a bigger nuclear to cytoplasmic ratio, nuclear pleomorphism as well as intranuclear inclusions. ${ }^{36}$ Furthermore, lymphocytes, neutrophils, macrophages as well as necrotic cells are also seen with very extreme vascularization. ${ }^{36-38}$ Some cells look like adipocytes because of existence of enormous lipomatous vacuoles and may makeup $5-10 \%$ of all the tumor cells and as high as $80 \%$ in single circumstances. ${ }^{36}$ Pseudopalisades are also seen and arranged from $30-1500 \mu \mathrm{m}$ in the greatest internal width and from 50-3500 $\mu \mathrm{m}$ in the greatest internal length with some areas showing the presence of multiple apoptotic cells.36,39 The malignancy risk of the lesion is associated with high degree of atypia, nuclear hyperchromatosis, amplified mitotic index, existence of areas of necrosis and atypical blood vessels. ${ }^{36}$

OLIG2 is a transcription factor associated with synthesis of oligodendrocytes and is well noted to partake in the initiation of malignant gliomas from neural stem cells. ${ }^{40,41}$ Recently, mutations of isocitrate dehydrogenase (IDH) genes have been linked to the pathogenesis glioma. ${ }^{42}$ IDH-1 and IDH-2 are NADP-dependent enzymes that catalyze the synthesis of $\alpha$-ketoglutarate from isocitrate during cellular metabolism. IDH-1 mutations are mostly seen concurrently with either TP53 mutations or co-deletion of $1 p / 19 q$ chromosomes which means that IDH-1 mutation partake actively in the pathogenesis of infiltrating gliomas. 42 Promoter methylation of methylguanineDNA methyltransferase (MGMT), co-deletion of $1 \mathrm{p} / 19 \mathrm{q}$ chromosomes, EGFR-phosphoinositide 3-kinase pathways and IDH-1 mutations have proven to be very crucial prognostic markers of GBM. 42 Ki-67 antigen appearance indicates the percentage of cellular and biological aggressiveness in malignancy.4,43,44 On the other hand, p53 protein is associated with the regulation of cell cycle and has been anomalous quantities of $\mathrm{p} 53$ protein is assumed to be related to amplified rates of proliferation. Therefore, mutations within the p53 gene frequently leads to anomalous secretion of p53 protein resulting in its buildup inside the nucleus of the cells.4,45 Alpha thalassemia/mental retardation syndrome Xlinked (ATRX) mutations has also been seen in diffuse GBM and linked to uncon- ventional lengthening of telomeres and appear to congregate with TP53 mutations within secondary GBM. 46,47

\section{Conclusions}

The diagnosis of intratumoral hemorrhage in our case was very challenging at the initial stages but with time the hematoma evolved into ring enhancing images typical of GBM. It's not every intracranial hematoma that is of pure vascular origin. The etiology of hemorrhage in our case although not well known is believed to be multifactorial. The management of our case was multidisciplinary incorporates surgery, radiotherapy as well as chemotherapy.

\section{References}

1. Liwnicz BH, Wu SZ, Tew Jr JM. The relationship between the capillary structure and hemorrhage in gliomas. J Neurosurg 1987;66:536-41.

2. Harada K, Kiya K, Matsumura S, et al. Spontaneous intracranial hemorrhage caused by oligodendroglioma: a case report and review of the literature. Neurol Med Chir (Tokyo) 1982;22:814.

3. Della Puppa A, Zustovich F, Gardiman $\mathrm{M}$, et al. Haemorrhagic presentation of low-grade glioma in adults. Acta Neurochir (Wien) 2007;149:1151-5.

4. Strojnik T. Prognostic Significance of Immunohistochemical Markers in Glioma Patients. Advances in the Biology, Imaging and Therapies for Glioblastoma: InTech; 2011.

5. Licata C, Turazzi S, Delfini R. Bleeding cerebral neoplasms with symptomatic hematoma/Comment. J Neurosurg Sci 2003;47:201.

6. Destian S, Sze G, Krol G, et al. MR imaging of hemorrhagic intracranial neoplasms. AJR Am J Roentgenol 1989;152:137-44.

7. Bitoh S, Hasegawa H, Ohtsuki H, et al. Cerebral neoplasms initially presenting with massive intracerebral hemorrhage. Surg Neurol 1984;22:57-62.

8. Padt J, De Reuck J, Vander Eecken H. Intracerebral hemorrhage as initial symptom of a brain tumor. Acta Neurol Belgica 1972;73:241-51.

9. Liebelt BD, Boghani Z, Takei H, et al. Epithelioid glioblastoma presenting as massive intracerebral hemorrhage: Case report and review of the literature. Surg Neurol Int 2015;6:S97.
10. Kondziolka D, Bernstein M, Resch L, et al. Significance of hemorrhage into brain tumors: clinicopathological study. J Neurosurg 1987;67:852-7.

11. Choi G, Park DH, Kang SH, Chung YG. Glioma mimicking a hypertensive intracerebral hemorrhage. J Korean Neurosurg Soc 2013;54:125-7.

12. Wakai S, Yamakawa K, Manaka S, Takakura K. Spontaneous intracranial hemorrhage caused by brain tumor: its incidence and clinical significance. Neurosurgery 1982;10:437-44.

13. Zimmerman RA, Bilaniuk LT. Computed tomography of acute intratumoral hemorrhage. Radiology 1980;135:355-59.

14. Can SM, Aydin Y, Turkmenoglu O, et al. Giant Cell Glioblastoma Manifesting as Traumatic Intracerebral Hemorrhage. Neurol medico-chirurgica. 2002;42:568-71.

15. Schrader B, Barth H, Lang EW, et al. Spontaneous intracranial haematomas caused by neoplasms. Acta Neurochir (Wien) 2000;142:979-85.

16. Watanabe K, Wakai S, Okuhata S. Gliomas presenting with basal ganglionic haemorrhage. Report of two cases. Acta Neurochir (Wien) 1997;139:787-8.

17. Davis ME. Glioblastoma: overview of disease and treatment. Clin J Oncol Nurs 2016;20:S2-8.

18. Young RM, Jamshidi A, Davis G, Sherman JH. Current trends in the surgical management and treatment of adult glioblastoma. Ann Transl Med 2015;3:121.

19. Richard SA, Liang RF, Lei CF, Liu YH. Diffuse leptomeningeal tuberculoma masquerading as leptomeningeal gliomatosis: a case report and review of literature. Infect Dis Trop Med 2017;3:e406.

20. Omuro A, DeAngelis LM. Glioblastoma and other malignant gliomas: a clinical review. JAMA 2013;310:1842-50.

21. Glantz MJ, Cole BF, Forsyth PA, et al. Practice parameter: anticonvulsant prophylaxis in patients with newly diagnosed brain tumors. Report of the Quality Standards Subcommittee of the American Academy of Neurology. Neurology 2000;54:1886-93.

22. Kothbauer $\mathrm{P}$, Jellinger K, Flament $\mathrm{H}$. Primary brain tumour presenting as spontaneous intracerebral haemorrhage. Acta Neurochirurgica 1979;49:35-45.

23. Nakayama Y, Tanaka A, Yoshinaga S, Ueno Y. [Indications for surgery to determine the etiology of subcortical hemorrhage]. No Shinkei Geka 
1998;26:1067-74.

24. Inamasu J, Kuramae T, Nakatsukasa M. Glioblastoma masquerading as a hypertensive putaminal hemorrhage: a diagnostic pitfall. Neurol Medico-chirurgica. 2009;49:427-9.

25. Broniscer A, Laningham FH, Kocak M, et al. Intratumoral hemorrhage among children with newly diagnosed, diffuse brainstem glioma. Cancer 2006;106:1364-71.

26. Meyer JR, Gorey MT. Differential diagnosis of nontraumatic intracranial hemorrhage. Neuroimaging Clin $\mathrm{N}$ Am 1998;8:263-93.

27. Atlas SW, Grossman RI, Gomori JM, et al. MR imaging of intracranial metastatic melanoma. J Comput Assist Tomography 1986;11:577-82.

28. Bodansky O. Methemoglobinemia and methemoglobin-producing compounds. Pharmacol Rev 1951;3:144-96.

29. Neill JM, Hastings AB. The influence of the tension of molecular oxygen upon certain oxidations of hemoglobin. J Biol Chem1925;63:479-92.

30. Gatenby R, Coia L, Richter M, et al. Oxygen tension in human tumors: in vivo mapping using CT-guided probes. Radiology 1985;156:211-4.

31. Harter D, Wilson T, Karajannis M. Glioblastoma multiforme: State of the art and future therapeutics. Surg Neurol Int 2014;5:64.

32. Zhao S, Wu J, Wang $C$, et al. Intraoperative fluorescence-guided resection of high-grade malignant gliomas using 5-aminolevulinic acid- induced porphyrins: A systematic review and meta-analysis of prospective studies. PloS One 2013;8:e63682.

33. Stupp R, Mason WP, van den Bent MJ, et al. Radiotherapy plus concomitant and adjuvant temozolomide for glioblastoma. $\mathrm{N}$ Engl $\mathrm{J}$ Med 2005;352:987-96.

34. Narayana A, Yamada J, Berry S, et al. Intensity-modulated radiotherapy in high-grade gliomas: clinical and dosimetric results. Int $\mathrm{J}$ Radiat Oncol Biol Phys 2006;64:892-7.

35. Barani IJ, Larson DA. Radiation therapy of glioblastoma. Current Understanding and Treatment of Gliomas: Springer; 2015. pp 49-73.

36. Urbańska K, Sokołowska J, Szmidt M, Sysa P. Glioblastoma multiforme-an overview. Contemp Oncol 2014; 18:307.

37. Schultz S, Pinsky GS, Wu NC, et al. Fine needle aspiration diagnosis of extracranial glioblastoma multiforme: Case report and review of the literature. Cytojournal 2005;2:19.

38. Linkous AG, Yazlovitskaya EM. Angiogenesis in glioblastoma multiforme: navigating the maze. Anticancer Agents Med Chem 2011;11:712-8.

39. Wilhelmsson U, Eliasson C, Bjerkvig R, Pekny M. Loss of GFAP expression in high-grade astrocytomas does not contribute to tumor development or progression. Oncogene 2003;22:3407.

40. Ballester LY, Wang Z, Shandilya S, et al. Morphologic characteristics and immunohistochemical profile of diffuse intrinsic pontine gliomas. Am J Surg Pathol. 2013;37:1357-1364.

41. Ligon KL, Huillard E, Mehta S, et al. Olig2-regulated lineage-restricted pathway controls replication competence in neural stem cells and malignant glioma. Neuron. 2007;53:503-517.

42. Lee KS, Choe G, Nam KH, et al. Immunohistochemical classification of primary and secondary glioblastomas. Korean J Pathol. 2013;47:541-548.

43. Scott RJ, Hall PA, Haldane JS, et al. A comparison of immunohistochemical markers of cell proliferation with experimentally determined growth fraction. J Pathol. 1991;165:173-178.

44. Wilson GD, Saunders MI, Dische S, et al. Direct comparison of bromodeoxyuridine and Ki-67 labelling indices in human tumours. Cell Prolif 1996; 29:141-52.

45. Cunningham JM, Kimmel DW, Scheithauer BW, et al. Analysis of proliferation markers and $\mathrm{p} 53$ expression in gliomas of astrocytic origin: relationships and prognostic value. J Neurosurg 1997;86:121-30.

46. Olar A, Aldape KD. Using the molecular classification of glioblastoma to inform personalized treatment. J Pathol 2014;232:165-77.

47. Jiao Y, Killela PJ, Reitman ZJ, et al. Frequent ATRX, CIC, FUBP1 and IDH1 mutations refine the classification of malignant gliomas. Oncotarget. 2012;3:709-22. 